\title{
Predicting Loss-of-Control Boundaries Toward a Piloting Aid
}

\author{
Jonathan S. Barlow* \\ Stinger-Gaffarian Technologies, NASA Ames Research Center, Moffett Field, CA, 94035, USA \\ Vahram Stepanyan ${ }^{\dagger}$ \\ Mission Critical Technologies, Inc., NASA Ames Research Center, Moffett Field, CA, 94035 \\ Kalmanje Krishnakumar ${ }^{\ddagger}$ \\ Intelligent Systems Division, NASA Ames Research Center, Moffett Field, CA, 94035
}

\begin{abstract}
This work presents an approach to predicting loss-of-control with the goal of providing the pilot a decision aid focused on maintaining the pilot's control action within predicted loss-of-control boundaries. The predictive architecture combines quantitative lossof-control boundaries, a data-based predictive control boundary estimation algorithm and an adaptive prediction method to estimate Markov model parameters in real-time. The data-based loss-of-control boundary estimation algorithm estimates the boundary of a safe set of control inputs that will keep the aircraft within the loss-of-control boundaries for a specified time horizon. The adaptive prediction model generates estimates of the system Markov Parameters, which are used by the data-based loss-of-control boundary estimation algorithm. The combined algorithm is applied to a nonlinear generic transport aircraft to illustrate the features of the architecture.
\end{abstract}

\section{Introduction}

I OsS-OF-CONTROL (LoC) has been the number one contributing factor to fatal airline accidents, and Lhas resulted in more fatalities than any other factor during the past ten years. ${ }^{1}$ Generally, LoC is characterized $^{2}$ as motion that is:

- outside the normal operating flight envelopes

- not predictably altered by pilot control inputs

- characterized by nonlinear effects, such as kinematic/inertial coupling, disproportionately large responses to small state variable changes, or oscillatory/divergent behavior

- likely to result in high angular rates and displacements

- characterized by the inability to maintain heading, altitude, and wings-level flight.

This work presents an approach to predicting LoC with the goal of providing the pilot a decision aid focused on maintaining the pilot's control action within predicted LoC boundaries. The predictive architecture estimates LoC boundaries by combining quantitative LoC criteria, a data-based predictive control boundary estimation algorithm and an adaptive prediction method.

\footnotetext{
*Senior Aerospace Research Engineer, SGT inc., NASA Ames Research Center, Mail Stop 269-1 P.O. Box 1 Moffett Field, CA 94035-1000, AIAA Member.

${ }^{\dagger}$ Research Scientist, Mission Critical Technologies, Inc., NASA Ames Research Center, Moffett Field, CA, 94035, AIAA Senior Member.

${ }^{\ddagger}$ Group Lead, Intelligent Systems Division, NASA Ames Research Center, Moffett Field, CA, 94035, AIAA Associate Fellow.
} 

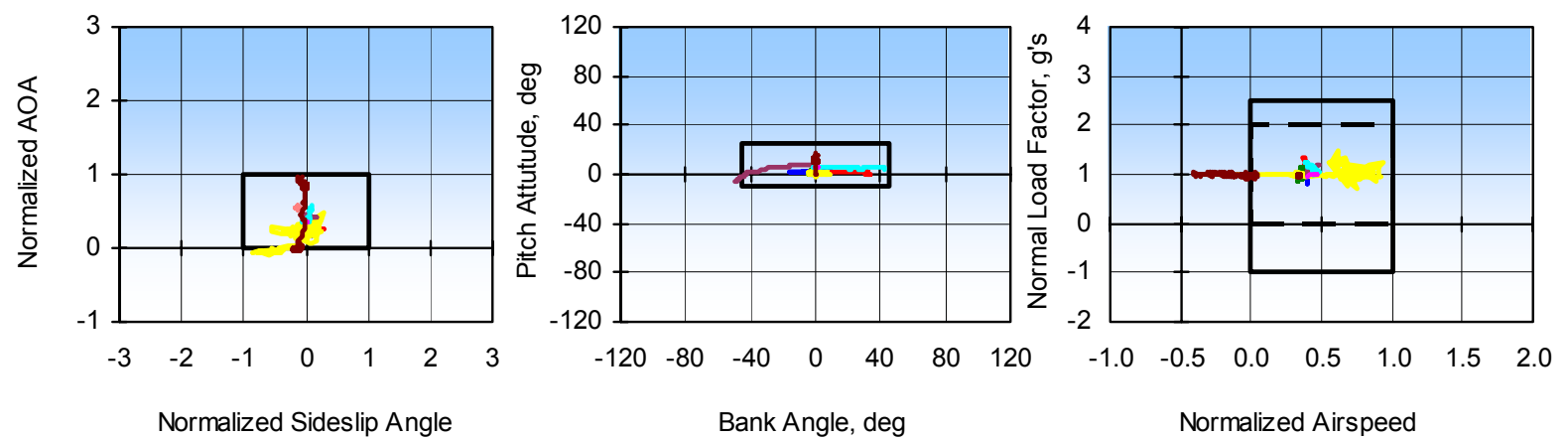

Normalized Sideslip Angle
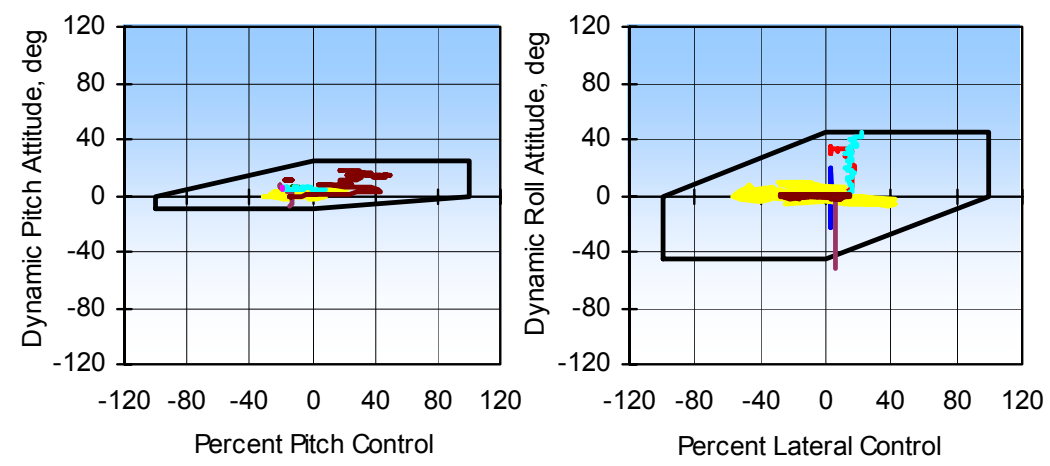

Normalized Airspeed

LEGEND

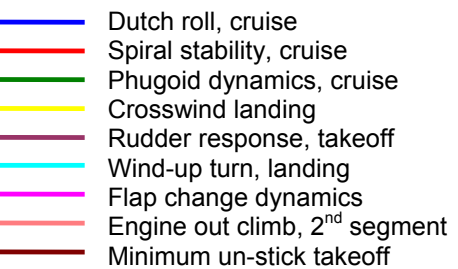

Figure 1. Quantitative LoC Criteria and flight data from Ref. 2.

Historically, LoC has been determined to be a factor in an accident by qualitative judgement based upon accident investigation experience. Quantitative $\mathrm{LoC}$ criteria $^{2}$ have been created to define LoC events. These criteria are in the form of boundary boxes on combinations of system states and control inputs, as seen in Figure 1. These boundaries are based on a historical LoC events. The authors propose that violation of any three of the quantitative LoC criteria constitutes LoC, violation of any two boundaries is borderline LoC, and typical aggressive flight test maneuvers typically violate at most one boundary. Our work presented in this paper combines multiple of the Quantitative LoC criteria into a single composite predictive boundary that aids the pilot in avoiding LoC scenarios. Particularly, the application in section V combines elements of the Pitch Attitude, Bank Angle, Dynamic Pitch Attitude, Dynamic Roll Attitude, Percent Pitch Control, and Percent Lateral Control criteria into a one two-dimensional display (discussed in Section II.A).

The LoC boundary estimation algorithm ${ }^{3}$ uses a data-based predictive control (DPC) approach to estimating boundaries on control actions to provide information to pilots and/or control systems to assist in avoiding LoC scenarios. The quantitative LoC criteria are used to define safe operating envelopes for the aircraft. A DPC algorithm was developed that finds the minimum control input that would result in the aircraft exceeding a safe operating envelope at various minimum time estimates. The calculated control inputs become a boundary of a set of safe control inputs. With this information, a pilot could change flying strategy or an autonomous system could schedule controller gains to prevent the vehicle from exceeding the envelope. The DPC algorithm assumes the aircraft is operating in the normal operating envelope, with approximately linear dynamics, and knowledge of linearized aircraft dynamics is available in the form of Markov Parameters.

The adaptive prediction model ${ }^{4}$ uses an adaptive prediction method for detection and mitigation of LoC, which works parallel to the flight control system without interacting with it. It takes the aircraft input and state measurement signals to generate the adaptive estimates of the unknown parameters, without requiring parameter convergence or special maneuvers. The estimated parameters are used to derive estimates of the system Markov Parameters. While the parameters of the adaptive prediction model do not converge to the parameters of the true system, the dynamics of the prediction model match the true dynamics of the system.

This work combines the DPC boundary estimation algorithm with the adaptive prediction method to estimate control boundaries for a nonlinear aircraft. The adaptive prediction model is used to generate 
estimates of the true Markov Parameters, which are used by the DPC estimation algorithm. The combined algorithm is applied to a nonlinear generic transport aircraft to illustrate the features of the architecture. The aircraft model includes nonlinear aircraft dynamics, actuator position and rate saturation, and surface failures.

\section{Pilot Decision Aid and the Predictive Architecture}

\section{A. Pilot Decision Aid}

The safest course in aircraft flight is to operate as far from the edge of the envelope as possible. However, maneuvering near the edge of the envelope may be required in certain circumstances (e.g. recovering after upset, take-off and landing). Furthermore, uncertainties introduced into the system, such as through damage or failure, can change the boundaries of the envelope, and can make certain control inputs more likely to take the aircraft out of the envelope. Control limits can be estimated using data-based predictive control and various minimum time estimates. The control limits can be represented as an n-dimensional hypercube, where $\mathrm{n}$ is the number of control inputs. The region within the box can be treated as a safe region.

A concept 2-dimensional display for a system with lateral and longitudinal control inputs is shown in Figure 2. Two notional safe regions are shown for the minimum control input needed to exceed the envelope within 1 second and 5 seconds. The current control input is also shown as being within the green box. The control limits can also be updated periodically, even in real time, ${ }^{5}$ as the limits change. This information may provide lead time for a pilot to change flying strategy or for an autonomous system to schedule controller gains to prevent the vehicle from exceeding the envelope.

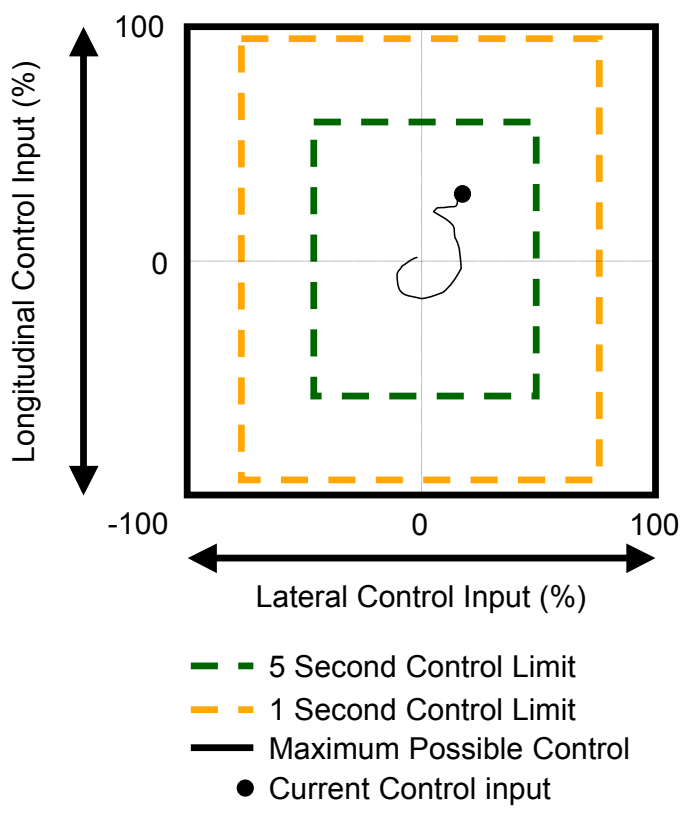

Figure 2. Conceptual 2-dimensional control limit display.

\section{B. Predictive Architecture for LoC Boundary Estimation}

The predictive architecture is shown in Figure 3. The black boxes and signal form the standard aircraft control architecture, and are unmodified for this work. The predictive architecture includes the the red boxes, which are the Adaptive Prediction Model, the DPC Law, the LoC Boundary Estimates, and the Pilot Aid. The predictive architecture uses data available from the standard architecture, including pilot command $r(t)$, input to the aircraft $u(t)$, and available states from the aircraft $x(t)$. The adaptive prediction model uses the available data to produce estimates system dynamics, and the DPC laws use the data and the estimated dynamics to predict LoC input boundaries, which are then fed to the piloting aid. The predictive architecture does not directly feed back commands or signals to the standard architecture. Indirect feedback is present only though the pilot, as the piloting aid may be used by the pilot to make decisions and take corrective action.

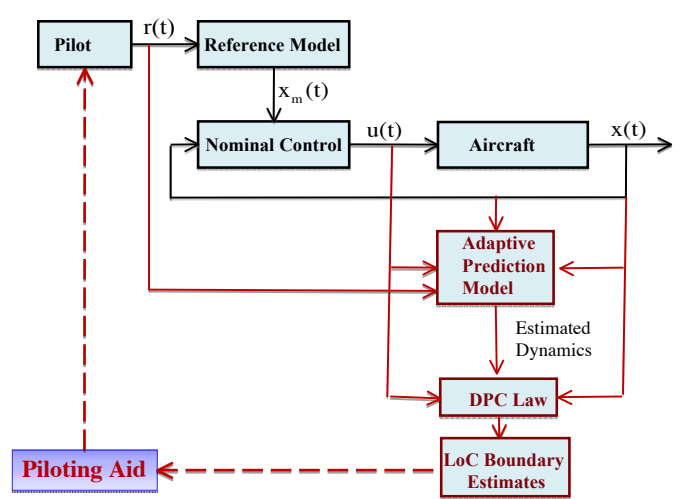

Figure 3. Predictive Architecture for LoC Boundary Estimation. 


\section{Adaptive Prediction Model}

In the previous section, knowledge of $S$, or an estimate

of $S$ is required for the identification of controller gains $G, H, K$. Although a nominal $S$ can be assumed and errors in the prediction can be compensated for by the identification of the controller gains, experience has shown that improved accuracy in $S$ leads to improved performance of the estimation. An adaptive prediction model $^{4}$ can be used to obtain estimates of the system dynamics. The adaptive prediction model is preferred over standard parameter estimation techniques as it does not require persistence of excitation for parameter convergence.

\section{A. Prediction Model}

The adaptive prediction model assumes knowledge of a nominal model $A_{0}, B_{0}$ of the system, where $A_{c}=$ $A_{0}+\Delta A$ and $B_{c}=B_{0}+\Delta B . A_{c}$ and $B_{c}$ are the continuous system matrices and $\Delta A$ and $\Delta B$ are the difference between the assumed system dynamics and the true system dynamics. The continuous system dynamics are expressed as

$$
\dot{x}(t)=A_{0} x(t)+B_{0} u(t)+\Delta A x(t)+\Delta B u(t)
$$

The prediction model is defined as

$$
\dot{\hat{x}}(t)=A_{0} \hat{x}(t)+B_{0} u(t)+\Delta \hat{A}(t) x(t)+\Delta \hat{B}(t) u(t)+\lambda(x(t)-\hat{x}(t))
$$

where $\lambda$ is a design parameter and $\Delta \hat{A}(t), \Delta \hat{B}(t)$ are the estimated parameters, updated by

$$
\begin{aligned}
\Delta \dot{\hat{A}}(t) & =\gamma \tilde{x} x^{T}(t) \\
\Delta \dot{\hat{B}}(t) & =\gamma \tilde{x} u^{T}(t)
\end{aligned}
$$

where $\tilde{x}=x(t)-\hat{x}(t)$ is the prediction error. The asymptotic behavior of the the prediction error, as well as the parameter errors defined by $\Delta \tilde{A}(t)=\Delta A(t)-\Delta \hat{A}(t), \Delta \tilde{B}(t)=\Delta B(t)-\Delta \hat{B}(t)$ is such that $\tilde{x} \rightarrow 0, \Delta \tilde{A}(t) \rightarrow 0, \Delta \tilde{B}(t) \rightarrow 0$ as $t \rightarrow \infty$. Further details of the derivation, theorems and proof of error properties can be found in Ref. 4 .

\section{Data-Based Predictive Control}

Consider an $n_{i}$-input, $n_{o}$-output system with the system state $x(k)$ and output $y(k)$ given by

$$
\begin{aligned}
x(k+1) & =A x(k)+B u(k)+B_{d} u_{d}(k) \\
y(k) & =C x(k)+D u(k)
\end{aligned}
$$

We assume that neither the system model, defined by $A, B, B_{d}, C$, and $D$, nor the initial state of the system, $x(0)$, are known, but a sufficiently rich and long excitation input $u(k)$ and possibly disturbance

corrupted output data $y(k)$ is available. The disturbance input, $u_{d}(k)$, if present, is assumed to be a sum of a finite number of unknown harmonics. Only an upper bound of the number of harmonics is known.

\section{A. Data-Based Predictive Control Law}

A predictive controller can be designed to minimize the receding-horizon cost function

$$
\begin{aligned}
J(k)= & {\left[y_{s}(k+\omega)-z_{s}(k+\omega)\right]^{T} Q\left[y_{s}(k+\omega)-z_{s}(k+\omega)\right]+} \\
& u_{s+\omega}^{T}(k) R u_{s+\omega}(k) \\
& +\left[u_{s+\omega}(k)-u_{s+\omega}(k-1)\right]^{T} R_{S}\left[u_{s+\omega}(k)-u_{s+\omega}(k-1)\right]
\end{aligned}
$$


where

$$
z_{s}(k+\omega) \equiv\left[\begin{array}{c}
z(k+\omega) \\
z(k+\omega+1) \\
\vdots \\
z(k+\omega+s-1)
\end{array}\right], y_{s}(k+\omega) \equiv\left[\begin{array}{c}
y(k+\omega) \\
y(k+\omega+1) \\
\vdots \\
y(k+\omega+s-1)
\end{array}\right], u_{s+\omega}(k) \equiv\left[\begin{array}{c}
u(k) \\
u(k+1) \\
\vdots \\
u(k+\omega+s-1)
\end{array}\right]
$$

The vector $z_{s}(k+\omega)$ is the desired output trajectory to be tracked. The output error cost is evaluated over the interval from time $k+\omega$ to $k+s+\omega-1$, with a weight matrix of $Q$. The control input cost is evaluated over the interval from time $k$ to $k+s+\omega-1$, with a weight matrix of $R$. The control smoothing cost, which is the weighted norm of the difference between the control input at time $k$ and the previous control input at time $k-1$, is evaluated over the interval from time $k$ to $k+s+\omega-1$, with weighting matrix $R_{S}$.

The predictive controller ${ }^{3}$ is in the dynamic feedback form:

$$
u(k)=G u_{\rho}(k-\rho)+H y_{\rho}(k-\rho)+K z_{s}(k+\omega)
$$

where

$$
u_{\rho}(k-\rho) \equiv\left[\begin{array}{c}
u(k-\rho) \\
u(k-\rho+1) \\
\vdots \\
u(k-1)
\end{array}\right], y_{\rho}(k-\rho) \equiv\left[\begin{array}{c}
y(k-\rho) \\
y(k-\rho+1) \\
\vdots \\
y(k-1)
\end{array}\right]
$$

In this context, $\rho$ is the number of past data points, $\omega$ is the start of the prediction window, and $s$ is the length of the prediction window. A conservative value for $\rho$ can be chosen using an upper bound on the order of the system and the number of distinct disturbance frequencies.

\section{B. Identification of Controller Gains}

To employ the optimal control law in Eq. 8, the controller gains $G, H, K$ must be either known a priori or estimated online. For an online implementation of the control laws, the gains are designed directly from input-output data via a relationship that relates $G, H$, and $K$ to input-output data. The direct relationship is the following equation:

$$
S u(k)=G u_{\rho}(k-\rho)+H y_{\rho}(k-\rho)+K y_{s}(k+\omega)
$$

where $S$ is the first $n_{i}$ rows of $\left\{I-\Gamma^{-1}\left[R+\left(I-L_{S}\right)^{T} R_{S}\left(I-L_{S}\right)\right]\right\}$ and $\Gamma$ is a combination of cost function weighting matrices and system parameters defined as

$$
\Gamma=\left(R+\left(I-L_{S}\right)^{T} R_{S}\left(I-L_{S}\right)+W^{T} Q W\right)
$$

where $W=\left[O_{s} C_{\omega}, T_{s}\right], C_{\omega}=\left[\begin{array}{llll}A^{\omega-1} B & \cdots & A B & B\end{array}\right]$,

$$
L_{S}=\left[\begin{array}{cccc}
0 & 0 & 0 & 0 \\
I_{r \times r} & 0 & 0 & 0 \\
0 & \ddots & 0 & 0 \\
0 & 0 & I_{r \times r} & 0
\end{array}\right], O_{s}=\left[\begin{array}{c}
C \\
C A \\
\vdots \\
C A^{s-1}
\end{array}\right] \text {, and } T_{s}=\left[\begin{array}{cccc}
D & & & \\
C B & D & & \\
\vdots & \ddots & \ddots & \\
C A^{s-2} B & \cdots & C B & D
\end{array}\right]
$$

Further detail about the derivation of Eq. 10 can be found in Ref. ${ }^{5}$ Eq. 10 has the property of being an open-loop input-output equation with the controller gains $G, H$, and $K$ from Eq. 8 included explicitly as coefficients of the equation. Using Eq. 10, the coefficients of the open-loop input-output model can be identified, and used in Eq. 8 as the gains of a dynamic feedback controller.

The data-based predictive controller developed for this study updates $G, H$, and $K$ using Eq. 10 and past input and output data. Since Eq. 10 is a non-causal input-output relationship, the approach begins 
with a time shift of $-(s+\omega)$ to the data sets within Eq. 10 in order to fully populate the super-vectors of collected data, with the most recent data used being $y(k-1)$ and $u(k-1)$. The time-shifted equation is then

$$
S u_{s+\omega}(k-s-\omega)=G u_{\rho}(k-\rho-s-\omega)+H y_{\rho}(k-\rho-s-\omega)+K y_{s}(k-s)
$$

Eq. 12 is then arranged in the form

$$
S u_{s+\omega}(k-s-\omega)=\Psi(k) \Phi(k-1)
$$

where

$$
\Psi(k)=[G(k) H(k) K(k)] \operatorname{and} \Phi(k-1)=\left[\begin{array}{c}
u_{\rho}(k-\rho-s-\omega) \\
y_{\rho}(k-\rho-s-\omega) \\
y_{s}(k-s)
\end{array}\right]
$$

In general, any linear estimation algorithm may be use to identify the parameters in $\Psi$. For this application a recursive least-squares estimation ${ }^{7}$ of the form

$$
\begin{gathered}
\Psi(k)=\Psi(k-1)+\{S u(k-s-\omega)-\Psi(k-1) \Phi(k-1)\} \frac{\Phi(k-1)^{T} \Theta(k-1)^{T}}{1+\Phi(k-1)^{T} \Theta(k-1) \Phi(k-1)} \\
\Theta(k)=\Theta(k-1)-\frac{\Theta(k-1) \Phi(k-1) \Phi(k-1)^{T} \Theta(k-1)^{T}}{1+\Phi(k-1)^{T} \Theta(k-1) \Phi(k-1)}
\end{gathered}
$$

is used to update $G, H$, and $K$ in $\Psi$, starting with some initially large covariance matrix $\Theta(0)$ and an initial guess of the controller gains $\Psi(0)$ and $S$. Obtaining an estimate of the parameter $S$ will be discussed in section D. In practice, the control error $\{S u(k-s-\omega)-\Psi(k-1) \Phi(k-1)\}$ found in Eq. 15 is subject to a dead-band. The estimation of $\Psi$ is conducted every time step. The optimal control law can be found as

$$
u(k)=G(k) u_{\rho}(k-\rho)+H(k) y_{\rho}(k-\rho)+K(k) z_{s}(k+\omega)
$$

for a set of desired outputs $z_{s}(k+\omega)$.

\section{Selection of Controller Parameters}

The control law in Eq. 8 and the input-output model in Eq. 10 are based on the interaction matrix formulation. ${ }^{6}$ To guarantee existence of the interaction matrix, the value of $\rho$ is selected such that $n_{o} \rho=n+2 f+1$ and $0 \leq \omega \leq \rho$, where $n$ is the system order, $f$ is the number of distinct disturbance frequencies, and the 1 accounts for a constant disturbance if present.

To determine the control boundary, the goal is to find the minimum control input that will result in the vehicle being at the boundary at a specified time $t$. Rather than specify an entire desired output trajectory $z_{s}(k+\rho)$ from the current time until time $t$, the start $\rho$ of the prediction window can be chosen as $\rho=t$, and the length $s$ of the prediction window can be chosen as $s=1$. Choosing the weighting matrix $Q$ to be a diagonal matrix with non-zero elements only for the $k+t$ time step can produce the same effect, but results in much more computation in practice. Including only the output error cost and control input cost results in a first control input $u(k)$ that is very conservative, and not representative of the boundary of the control. By including the control smoothing cost, we can cause the control input to be nearly constant from the current time until the end of the prediction window. Also, by setting the first $n_{i}$ diagonal elements of $R_{S}$ to zero, the controller is allowed to select any control input, and afterwards remain nearly constant. Setting the first $n_{i}$ diagonal elements of $R_{S}$ to zero also has the benefit of eliminating an additional term from the control law in Eq. 8 that would otherwise be present, thus simplifying the computation of the control input. The resulting control is the amplitude of a step input that, if applied, would result in the vehicle being at the boundary of the envelope at time $t$. It is important to note that an under-damped second order system will have overshoot, and for a time $t$ that is chosen beyond the peak overshoot, the system will leave the envelope before $t$, and return to the boundary at time $t$. Therefore, the boundary generated is an estimate, not a guarantee of the minimum control that will result in the vehicle leaving the envelope. However, multiple algorithms each having a unique time estimate $t$ can be used to create multiple limits, and the innermost box could be considered the safe region of operation. 


\section{Integration with Adaptive Prediction Model}

The prediction model from section III is to be used with the data-based predictive control (DPC) algorithm to generate the estimated control input boundaries. The DPC algorithm expects discrete $A$ and $B$ matrices of the form shown in Eq. 5. Estimates of the continuous system matrices $\hat{A}_{c}, \hat{B}_{c}$ are formed using the estimated parameters from Eq. 2 as $\hat{A}_{c}(t)=A_{0}+\Delta \hat{A}(t), \hat{B}_{c}(t)=B_{0}+\Delta \hat{B}(t)$. The matrices $\hat{A}_{c}, \hat{B}_{c}$ are fixed over the future horizon for which the DPC algorithm is designed, and $\hat{A}_{c}, \hat{B}_{c}$ are discretized and used to compute $\Gamma$ using Eq. 11. $\Gamma$ can then be used to compute an estimate of $\mathrm{S}$ as the first $n_{i}$ rows of

$\left\{I-\Gamma^{-1}\left[R+\left(I-L_{S}\right)^{T} R_{S}\left(I-L_{S}\right)\right]\right\}$, and $\mathrm{S}$ can be used in Eq. 15 to update the control gains $\Psi(k)$. The gains $\Psi(k)$ are then used to compute the boundary using Eq. 17.

\section{Application to a Non-linear Generic Transport Model}

\section{A. Generic Transport Model}

The operation of the Data-Based Predictive Control algorithm with Adaptive Prediction model is demonstrated with a medium fidelity 6-DOF generic transport model ${ }^{8}$ (GTM) with nonlinear coupled aerodynamics, airframe damage effects, and actuator position and rate saturation limits.

\section{B. Experimental Set-up}

The GTM model is trimmed at Mach 0.8 and 30k feet. Both a nominal aircraft and an off-nominal aircraft are simulated for comparison. The off-nominal aircraft has a $25 \%$ tip loss of the left elevator. Both aircraft are trimmed at the start of the simulation, and receive the same pre-recorded inputs.

For this example, a single DPC algorithm is designed for the entire aircraft. The order of the system is $n=9$, the number of outputs $n_{i}$ is 2 , and there are no disturbances, so the minimum $\rho$ that can be used is 5. If only an estimate of the order of the system and an upper bound on the number of disturbance frequencies is known, then $\rho$ can be chosen conservatively. For this example, $\rho$ is selected to be 10 as such a conservative choice. As discussed previously, the length $s$ of the prediction window is selected as $s=1$, and the values for $\omega$ used correspond to 3 seconds, or $\omega=300$ for a sampling period of $d t=0.01$, which is used for this example. The output error weighting matrix $Q$ is a single value and chosen to be $Q=1$, and the control input weighting matrix $R$ is a diagonal matrix with diagonal elements 0.000001 . The control smoothing weighting matrix $R_{S}$ is a diagonal matrix with first two diagonal values 0 , and subsequent values 10. An initial guess for controller gains $\mathrm{G}, \mathrm{H}$, and $\mathrm{K}$ were computed from linear decoupled state-space aircraft models at a different trim point. Both the nominal and off-nominal systems were then simulated with the same pre-recorded sequence of pilot inputs applied to each system.

\section{Results}

Figures 4 and 5 show a series of snapshots of the control inputs and control limits during six seconds of simulation. A two-second trace of the control history is shown as a continuous black line, with the earliest point marked as a black dot. The boundaries shown are also the boundaries for the time at which the snapshot was taken. The aircraft attitude is also shown for one second leading up to the time at which the snapshot was taken.

In the first column of Figure 4, the aircraft has maneuvered from steady-level flight to a right-banked turn, and the reduced longitudinal control boundary for the off-nominal condition is clearly visible. The control boundary for the off-nominal aircraft is less than the control boundary for the nominal aircraft. The lateral boundary has saturated at full left lateral control input, thus indicating that the aircraft will not leave the lateral boundary within three seconds. In the second column of Figure 4, the aircraft is rolling to the left, and the off-nominal lateral boundary is now less than full lateral control input, due to the asymmetric tail loss. The first column of Figure 5 shows the condition as the aircraft rolls back to the right, showing the reduced control boundary and indicating that the aircraft can still exit the lateral boundary. The second column of Figure 5 shows the condition as the aircraft rolls back to the wings level, saturated lateral control boundary. 


\section{Conclusion}

This work extends previous results for estimation of control boundaries that permit safe operation of an aircraft to uncertain and linear time-varying systems. This work is envisioned to assist pilots or autonomous systems in predicting and preventing LoC events. The algorithm is based on input-output data, and the boundaries can be updated for the current aircraft and flight condition. The control boundaries may help to reduce inappropriate pilot actions, and may help alert the pilot to reduced control authority.

\section{References}

\footnotetext{
${ }^{1}$ Statistical Summary of Commercial Jet Airplane Accidents Worldwide Operations 1959-2009, p. 23, Aviation Safety, Boeing Commercial Airplanes, Seattle, WA, USA, 2010.

${ }^{2}$ Wilborn, J. E. and Foster, J. V., Defining Commercial Transport Loss-of-Control: A Quantitative Approach, Proceedings of the AIAA Flight Mechanics Conference and Exhibit, Providence, RI, August 2004.

${ }^{3}$ Barlow, J., Stepanyan, V. and Krishnakumar, K., Estimating Loss-of-Control: a Data-Based Predictive Control Approach, AIAA Guidance, Navigation, and Control Conference, Portland, Oregon, August 2011.

${ }^{4}$ Stepanyan, V., Krishnakumar, K., Barlow, J., and Bjil, H., Adaptive Estimation Based Loss of Control Detection and Mitigation, AIAA Guidance, Navigation, and Control Conference, Portland, Oregon, August 2011.

${ }^{5}$ Barlow, J. S., and Phan, M. Q., Direct Data-Based Model Predictive Controller Design, AIAA Guidance, Navigation, and Control Conference, Keystone, Colorado, August 2006.

${ }^{6}$ Darling, R. S. and Phan, M. Q., Dynamic Output Feedback Predictive Controllers for Vibration Suppression and Periodic Disturbance Rejection, Proceedings of the 14th AAS/AIAA Space Flight Mechanics Meeting, Maui, HI, February 2004.

${ }^{7}$ Goodwin, G.C. and Sin, K.S., Adaptive Filtering Prediction and Control, Prentice-Hall, Englewood Cliffs, NJ, 1984.

${ }^{8}$ Nguyen, N., Krishnakumar, K., Kaneshige, J., and Nespeca, P., "Flight Dynamics Modeling and Hybrid Adaptive Control of Damaged Asymmetric Aircraft," AIAA Journal of Guidance, Control, and Dynamics, Vol. 31, No. 3, 2008, pp. 751-764
} 


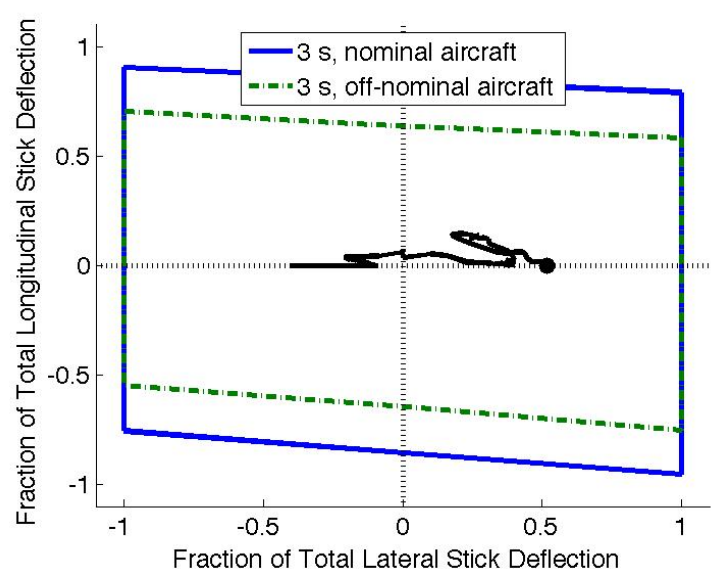

(a) Boundary $\mathrm{t}=1 \mathrm{~s}$

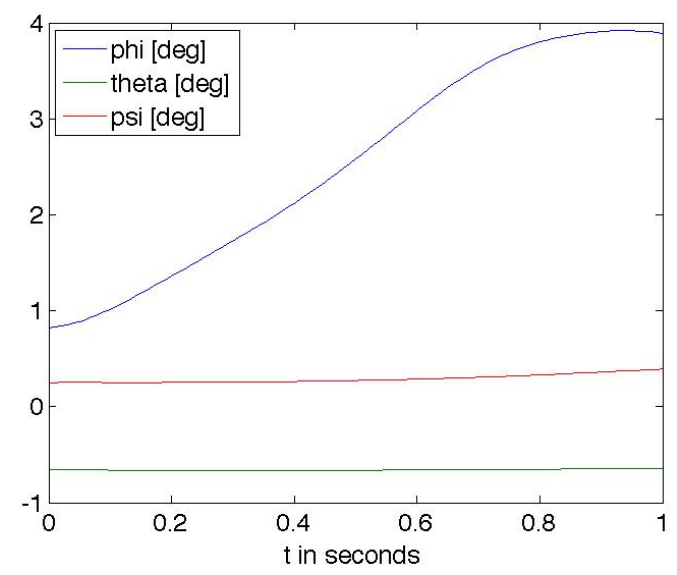

(c) Attitude in degrees

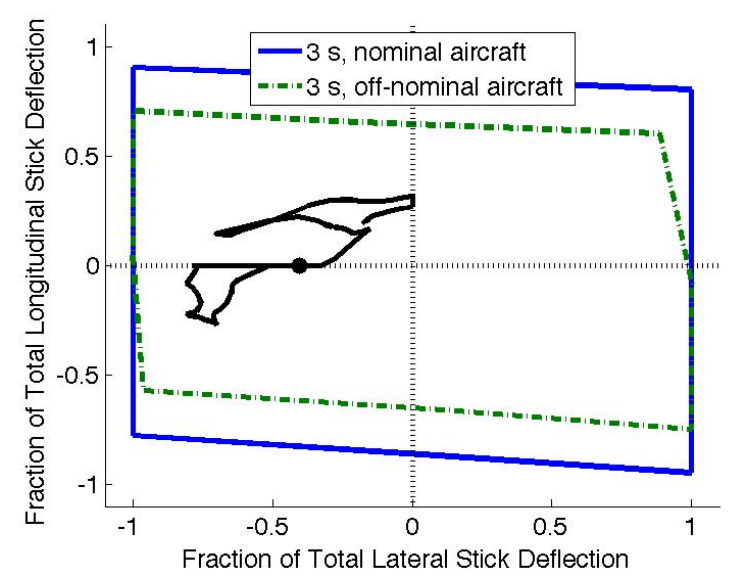

(b) Boundary $\mathrm{t}=2 \mathrm{~s}$

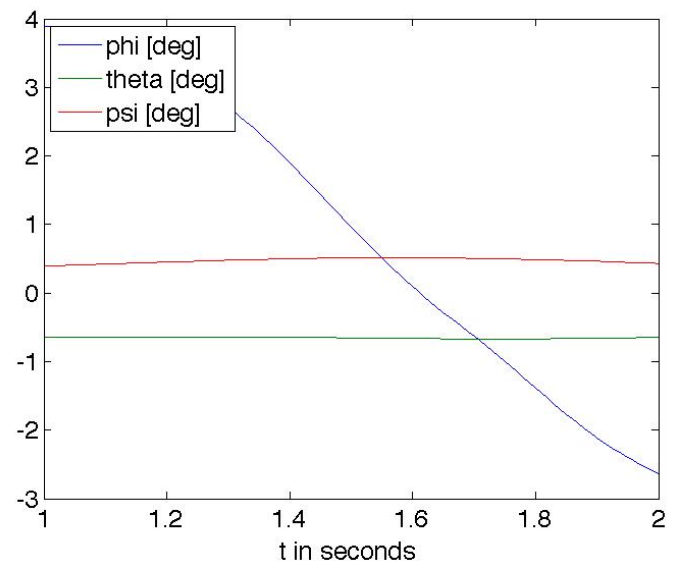

(d) Attitude in degrees

Figure 4. Time series of boundaries and aircraft attitude at $t=1 \mathrm{~s}$ and $t=2 \mathrm{~s}$. 


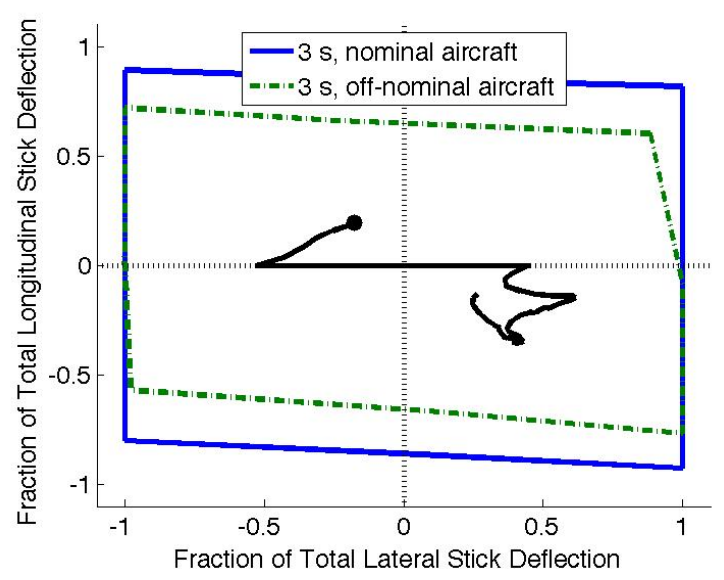

(a) Boundary $t=3 \mathrm{~s}$

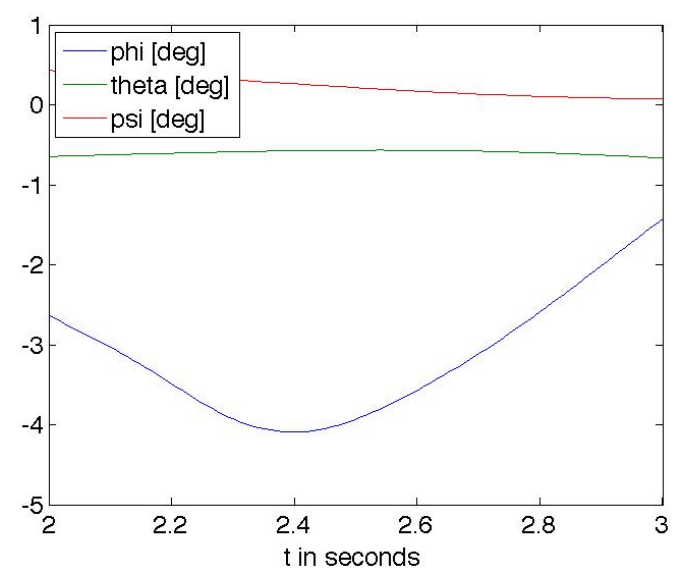

(c) Attitude in degrees

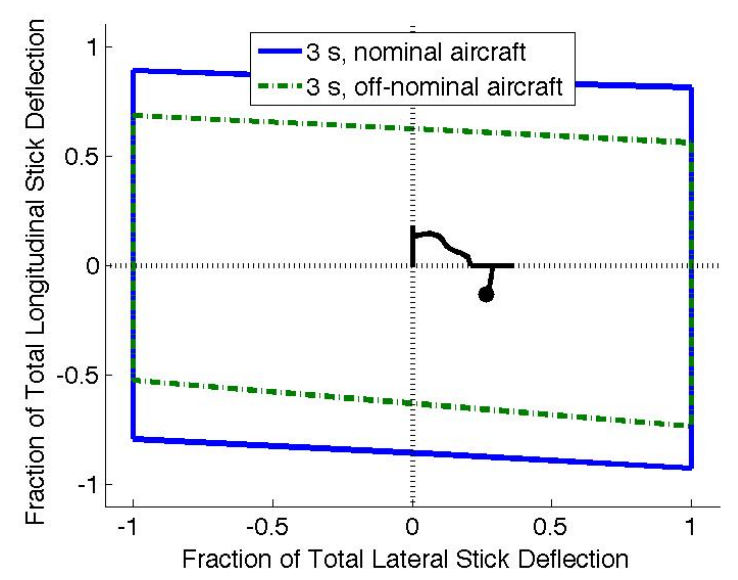

(b) Boundary $\mathrm{t}=4 \mathrm{~s}$

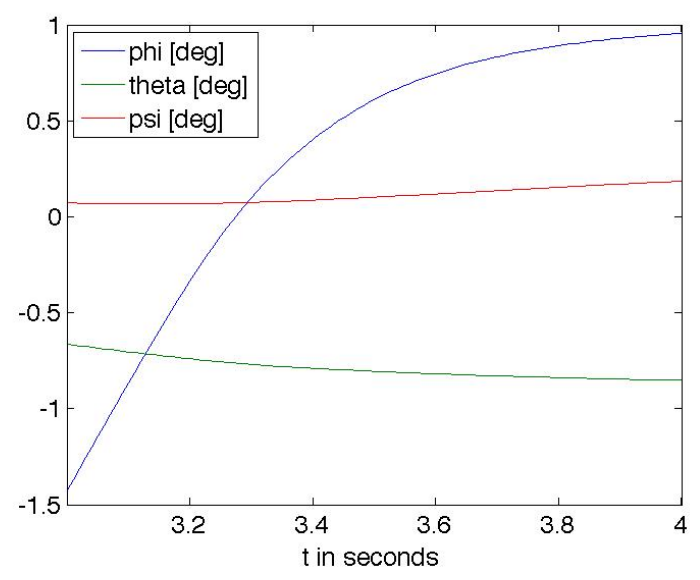

(d) Attitude in degrees

Figure 5. Time series of boundaries and aircraft attitude at $t=3 \mathrm{~s}$ and $t=4 \mathrm{~s}$. 